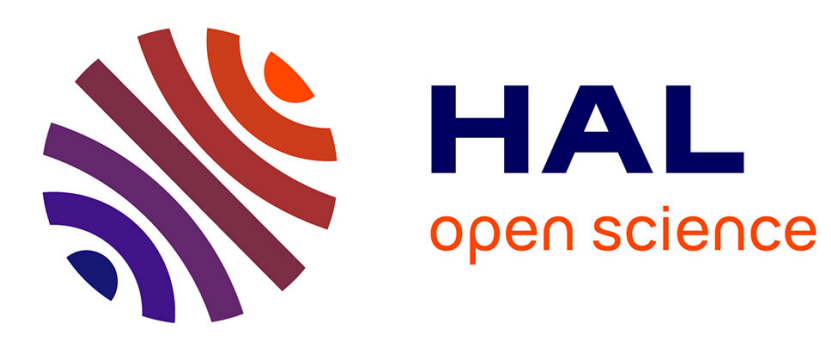

\title{
Croissance épitaxique en phase liquide de GaxIn1-xp
}

\author{
H. Mariette, J. Bourneix, A. Marbeuf
}

\section{To cite this version:}

H. Mariette, J. Bourneix, A. Marbeuf. Croissance épitaxique en phase liquide de GaxIn1-xp. Revue de Physique Appliquée, 1976, 11 (4), pp.475-481. 10.1051/rphysap:01976001104047500 . jpa-00244082

\section{HAL Id: jpa-00244082 https://hal.science/jpa-00244082}

Submitted on 1 Jan 1976

HAL is a multi-disciplinary open access archive for the deposit and dissemination of scientific research documents, whether they are published or not. The documents may come from teaching and research institutions in France or abroad, or from public or private research centers.
L'archive ouverte pluridisciplinaire HAL, est destinée au dépôt et à la diffusion de documents scientifiques de niveau recherche, publiés ou non, émanant des établissements d'enseignement et de recherche français ou étrangers, des laboratoires publics ou privés. 
Classification

Physics Abstracts

9.118

\title{
CROISSANCE ÉPITAXIQUE EN PHASE LIQUIDE DE $\mathbf{G a}_{x} \mathrm{In}_{1-x} \mathbf{P}$
}

\author{
H. MARIETTE, J. BOURNEIX et A. MARBEUF \\ Laboratoire de Physique des Solides, C. N. R. S., 1, Place A. Briand 92190, Meudon Bellevue, France
}

(Reçu le 9 février 1976, révisé le 22 mars 1976, accepté le 22 mars 1976)

\begin{abstract}
Résumé. - La détermination des chemins de cristallisation dans le diagramme d'équilibre Ga-In-P permet de prévoir les conditions de croissance de couches épitaxiques homogènes $\mathrm{Ga}_{x} \mathrm{In}_{1-x} \mathrm{P}$. Des dépôts monocristallins sur substrats de GaP orientés suivant le plan (111) $\mathrm{B}$ ont été préparés pour $x>0,80$ par refroidissement d'une solution saturée. Des couches de bonne qualité sur supports de GaAs orientés (111) B ont été obtenues dans le domaine de composition $0,50<x<0,52$ par une méthode verticale de croissance épitaxique en phase liquide à température constante. Les spectres de luminescence caractéristiques de ces dépôts sont présentés.
\end{abstract}

\begin{abstract}
The determination of crystallization paths in the phase diagram of Ga-In-P allows us to predict the growth condition for of homogeneous epitaxial films of $\mathrm{Ga}_{x} \operatorname{In}_{1-x} \mathrm{P}$. Monocrystalline layers were obtained on (111) B oriented GaP substrates for $x>0.80$ by cooling of the saturated melt. Layers of good quality have been grown on (111) B oriented GaAs substrates for $0.50<x<0.52$ using a constant temperature liquid phase epitaxial vertical process. The typical luminescence spectra of these layers are presented.
\end{abstract}

1. Introduction. - Le système ternaire $\mathrm{Ga}_{x} \mathrm{In}_{1-x} \mathrm{P}$ présente un grand intérêt potentiel en vue des dispositifs électroluminescents [1]. En effet, par suite du croisement des bandes de conduction $\Gamma$ et $\mathrm{X}$, il offre à la fois des semiconducteurs à transition directe pour $x<0,70$ et à transition indirecte lorsque $x>0,70$; en outre, la largeur de bande interdite au point de croisement est particulièrement élevée $(2,2 \mathrm{eV}$ environ à $300 \mathrm{~K})$ [2-6]. Cependant la réalisation de composants électroniques avec ces alliages est limitée par la grande difficulté d'obtention de monocristaux. Après de nombreux travaux qui ont permis de déterminer les propriétés fondamentales des alliages $\mathrm{Ga}_{x} \mathrm{In}_{1-x} \mathrm{P}$, de nouvelles recherches tendant à obtenir des matériaux de bonne qualité cristalline sont nécessaires.

En effet, par suite de la différence importante entre les paramètres de réseau de $\mathrm{GaP}(a=5,451 \AA)$ et de InP $(a=5,869 \AA)$, une très légère variation de composition dans l'alliage entraîne des contraintes importantes ; il s'ensuit une grande densité de dislocations, voire même l'obtention de polycristaux. Il faut donc pouvoir développer une croissance localement très uniforme en composition.

Partant de la connaissance précise du diagramme d'équilibre ternaire dans la région riche en indium, en utilisant un modèle de solution pseudo-régulière, nous présentons ici une étude des chemins de cristallisation qui permettent de prévoir l'évolution de la composition de l'alliage formé en fonction de l'abaissement de la température de croissance.
Suivant la composition désirée de la solution solide, nous sommes amenés à utiliser deux méthodes de croissance différentes ; de plus, afin de réaliser ces alliages sous forme de couches épitaxiées, deux types de substrat doivent être choisis pour minimiser le désaccord paramétrique entre le substrat et la couche :

- Dans le cas d'alliages $\mathrm{Ga}_{\boldsymbol{x}} \mathrm{In}_{1-x} \mathrm{P}$ de composition $x$ comprise entre 0,4 et 0,6 , une cristallisation à température constante s'impose pour obtenir des couches relativement épaisses et très homogènes. Ces dépôts sont effectués sur des substrats de GaAs orientés (111) B. Ce matériau a été choisi en raison de son paramètre de réseau $(a=5,65 \AA ̊)$ égal à celui d'un alliage de composition $x=0,51$ [7]. Nous avons donc mis au point une technique verticale de croissance épitaxique en phase liquide à température constante.

- Pour les alliages de composition $x$ riches en $\mathrm{GaP}$ $(x \geqslant 0,8)$ nous avons choisi des substrats de $\overline{\mathrm{GaP}}$ orientés (111) B. Les dépôts ont été élaborés à partir de la technique ci-dessus, et aussi à partir d'une méthode classique d'épitaxie horizontale en phase liquide avec abaissement progressif de la température de cristallisation.

Nous avons caractérisé l'homogénéité et la qualité cristalline des couches obtenues et nous présentons également leur spectre de luminescence à 4,2 K.

2. Considérations thermodynamiques. - 2.1 DiAGRAMME DE PHASE. - Les conditions thermodynami- 
TABLEAU I

Paramètres thermodynamiques

\begin{tabular}{|c|c|c|c|c|}
\hline & Ga-P & In-P & Ga-In & GaP-InP \\
\hline$\Omega\left(\mathrm{cal} . \mathrm{mole}^{-1}\right)$ & $2800-\overline{4,8} \mathrm{~T}[10]$ & $4600-4 \mathrm{~T}[10]$ & $10 \overline{66}[9]$ & 3200 \\
\hline $\begin{array}{c}\Delta S^{\mathrm{F}}(\underset{\text { u.e.mole }}{-1}) \\
T^{\mathrm{F}}(\mathrm{K})\end{array}$ & $\begin{array}{r}16,8 \\
1738\end{array}$ & $\begin{array}{c}14 \\
1343\end{array}$ & & \\
\hline
\end{tabular}

ques pour l'équilibre dans le système ternaire Ga-In-P peuvent être écrites [8] sous la forme :

$$
\begin{array}{r}
\ln \left(x_{\mathrm{GaP}}^{\mathrm{s}} \gamma_{\mathrm{GaP}}\right)=\ln \left(4 x_{\mathrm{Ga}}^{1} x_{\mathrm{P}}^{1} \gamma_{\mathrm{Ga}} \gamma_{\mathrm{P}} / \gamma_{\mathrm{Ga}}^{\mathrm{ISGaP}} \gamma_{\mathrm{P}}^{\mathrm{lsGaP}}\right)+ \\
+\Delta S_{\mathrm{GaP}}^{\mathrm{F}}\left(T_{\mathrm{GaP}}^{\mathrm{F}}-T\right) / R T \\
\ln \left(x_{\mathrm{InP}}^{\mathrm{s}} \gamma_{\mathrm{InP}}\right)=\ln \left(4 x_{\mathrm{In}}^{1} x_{\mathrm{P}}^{1} \gamma_{\mathrm{In}} \gamma_{\mathrm{P}} / \gamma_{\mathrm{In}}^{\mathrm{IIIP}} \gamma_{\mathrm{P}}^{\mathrm{IIISP}}\right)+ \\
+\Delta S_{\mathrm{InP}}^{\mathrm{F}}\left(T_{\mathrm{InP}}^{\mathrm{F}}-T\right) / R T
\end{array}
$$

avec

$$
\begin{array}{r}
x_{\mathrm{Ga}}^{1}+x_{\mathrm{In}}^{1}+x_{\mathrm{P}}^{1}=1 \\
x_{\mathrm{GaP}}^{\mathrm{s}}+x_{\mathrm{InP}}^{\mathrm{s}}=1
\end{array}
$$

$x_{\mathrm{GaP}}^{\mathrm{s}}$ et $x_{\mathrm{InP}}^{\mathrm{s}}$ sont les fractions molaires respectivement de GaP et de InP dans la solution solide GaP-InP, $\gamma_{\mathrm{GaP}}$ et $\gamma_{\mathrm{InP}}$ les coefficients d'activité dans la phase solide ; $x_{\mathrm{Ga}}^{1}, x_{\mathrm{In}}^{1}, x_{\mathrm{P}}^{1}, \gamma_{\mathrm{Ga}}, \gamma_{\mathrm{In}}, \gamma_{\mathrm{P}}$ représentent les fractions molaires et les coefficients d'activité dans la phase liquide, $\gamma_{G a}^{\text {lsGaP }}\left(\gamma_{\mathbf{P}}^{\text {lsGaP }}\right)$ sont les coefficients d'activité $d u$ $\mathrm{Ga}(\mathrm{P})$ dans un liquide de composition $x_{\mathrm{Ga}}^{1}=x_{\mathrm{P}}^{1}=0,5$; mêmes définitions pour $\gamma_{\ln }^{\operatorname{linP}}$ et $\gamma_{\mathbf{P}}^{\operatorname{ls} I n P}$.

Les températures et entropies de fusion de $\mathrm{GaP}$ et de InP sont notées $T^{\mathrm{F}}$ et $\Delta S^{\mathrm{F}}$.

Le modèle de solution pseudo-régulière décrit bien les solutions liquides III-V et fournit des coefficients d'activité qui dépendent de paramètres d'interaction $\Omega_{\mathrm{ij}}$ entre les atomes i et j :

$$
\begin{aligned}
& R T \ln \gamma_{\mathrm{Ga}}=\Omega_{\mathrm{Ga}-\mathrm{In}}\left(x_{\mathrm{In}}^{1}\right)^{2}+\Omega_{\mathrm{Ga}-\mathrm{P}}\left(x_{\mathrm{P}}^{1}\right)^{2}+ \\
&+\left(\Omega_{\mathrm{Ga}-\mathrm{P}}-\Omega_{\mathrm{In}-\mathrm{P}}+\Omega_{\mathrm{Ga}-\mathrm{In}}\right) x_{\mathrm{In}}^{1} x_{\mathrm{P}}^{1} \\
& R T \ln \gamma_{\mathrm{In}}=\Omega_{\mathrm{Ga}-\mathrm{In}}\left(x_{\mathrm{Ga}}^{1}\right)^{2}+\Omega_{\mathrm{In}-\mathrm{P}}\left(x_{\mathrm{P}}^{1}\right)^{2}+ \\
&+\left(\Omega_{\mathrm{In}-\mathrm{P}}-\Omega_{\mathrm{Ga}-\mathrm{P}}+\Omega_{\mathrm{Ga}-\mathrm{In}}\right) x_{\mathrm{Ga}}^{1} x_{\mathrm{P}}^{1} \\
& R T \ln \gamma_{\mathrm{P}}=\Omega_{\mathrm{Ga}-\mathrm{P}}\left(x_{\mathrm{Ga}}^{1}\right)^{2}+\Omega_{\mathrm{In}-\mathrm{P}}\left(x_{\mathrm{In}}^{1}\right)^{2}+ \\
&+\left(\Omega_{\mathrm{Ga}-\mathrm{P}}+\Omega_{\mathrm{In}-\mathrm{P}}-\Omega_{\mathrm{Ga}-\mathrm{In}}\right) x_{\mathrm{Ga}}^{1} x_{\mathrm{In}}^{1}
\end{aligned}
$$

Le même modèle peut être appliqué aux solutions solides et introduit un paramètre d'interaction supplémentaire :

$$
\begin{aligned}
& R T \ln \gamma_{\mathrm{GaP}}=\Omega_{\mathrm{GaP}-\mathrm{InP}}\left(1-x_{\mathrm{GaP}}^{\mathrm{s}}\right)^{2} \\
& R T \ln \gamma_{\mathrm{InP}}=\Omega_{\mathrm{GaP}-\mathrm{InP}}\left(1-x_{\mathrm{InP}}^{\mathrm{s}}\right)^{2} .
\end{aligned}
$$

Après avoir choisi une valeur pour chaque paramètre d'interaction, on peut résoudre l'ensemble des éq. (1) et (2) en se fixant pour chaque itération du calcul deux inconnues.

Le choix des paramètres utilisés est présenté dans le tableau I.

Le paramètre d'interaction $\Omega_{\mathrm{GaP}-\mathrm{InP}}$ a été déterminé pour être en bon accord avec les points expérimentaux obtenus comme décrit aux paragraphes 2 et 3 ci-après et représentés sur les figures 2 et 3 . La valeur obtenue $\Omega_{\mathrm{GaP}-\mathrm{InP}}=3200 \mathrm{cal}$. mole ${ }^{-1}$ est comparable à celle de K. Kajiyama (4000 cal.mole ${ }^{-1}[11]$ ), de M. B. Panish et M. Ilegems (3 $500 \mathrm{cal}^{-\mathrm{mole}^{-1}}$ [10]) et de L. M. Forster (3 250 cal.mole ${ }^{-1}$ [12]). Notre valeur est en bon accord avec celle de G. B. Stringfellow obtenue par un calcul semi-empirique à partir des enthalpies d'atomisation de GaP et de InP (3 $630 \mathrm{cal}^{\mathrm{mole}} \mathrm{m}^{-1}$ [13]). La figure 1 représente le diagramme ainsi obtenu

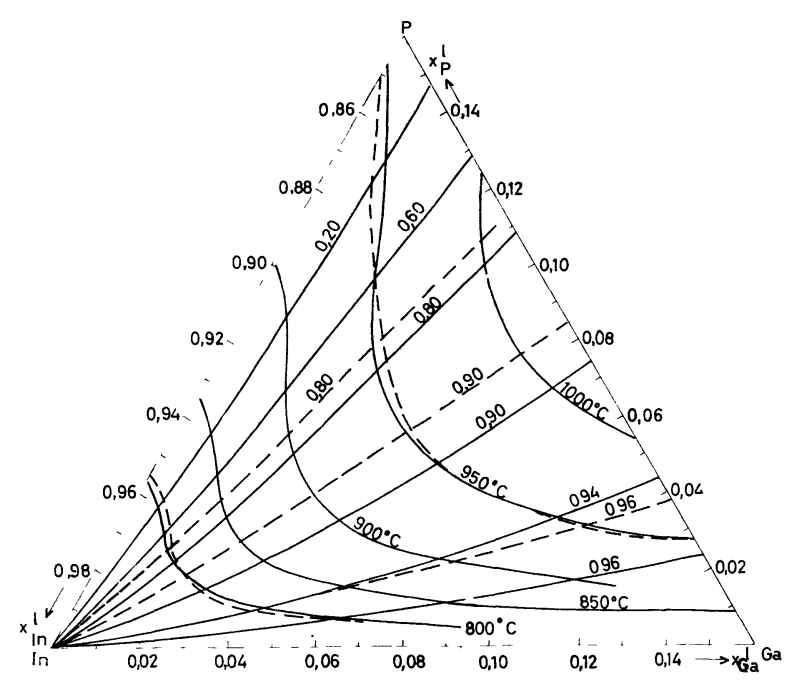

FIG. 1. - Diagramme de phase Ga-In-P dans la région riche en indium :- lignes isosolidus et liquidus calculées dans le modèle de solution pseudo-régulière (ce travail) ; - - - lignes isosolidus et liquidus calculées dans le modèle quasi chimique (G. B. Stringfellow [9]).

dans la région riche en indium et permet de le comparer avec celui de G. B. Stringfellow [3], [9].

2.2 Chemins De CRistallisation. - Notre but est de prévoir l'évolution de la composition du solide $\mathrm{Ga}_{x} \mathrm{In}_{1-x} \mathrm{P}$ obtenu lorsque l'on diminue lentement la température d'une solution liquide initialement saturée. Nous écrivons pour cela que, au cours d'un intervalle élémentaire de température $\delta T$ il y a conservation de 
masse : la quantité des solutés contenus dans le liquide à la température $T$ est égale à celle contenue dans le solide formé à $T-\delta T$ et le liquide restant.

Nous obtenons ainsi une nouvelle équation :

$\left(x_{G a}^{1}-x_{G a}^{10}\right) /\left(x_{G a}^{1}-0,5 x_{G a P}^{s}\right)=\left(x_{P}^{1}-x_{P}^{10}\right) /\left(x_{P}^{1}-0,5\right)$

où $x_{\mathrm{Ga}}^{1_{0}}$ et $x_{\mathrm{P}}^{1_{0}}$ représentent la composition de la phase liquide à la température $T, x_{\mathrm{Ga}}^{1}$ et $x_{\mathrm{P}}^{1}$ la composition après le refroidissement $\delta T ; x_{\mathrm{GaP}}^{\mathrm{s}}$ est la composition de la solution solide formée à la température $T-\delta T$.

Cette équation supplémentaire traduit l'hypothèse suivante faite sur le mécanisme de croissance : un refroidissement infiniment lent $\mathrm{du}$ bain permet de supposer, en première approximation, qu'à chaque instant le système est en équilibre ; ceci n'est vrai que si l'homogénéisation du liquide par diffusion et convection est beaucoup plus rapide que le refroidissement, en d'autres termes si la vitesse de cristallisation est inférieure à la vitesse de diffusion du gallium et du phosphore dans le liquide.

Compte tenu de cette hypothèse, la fraction de solide formé lors d'un refroidissement élémentaire $\delta T$ de la

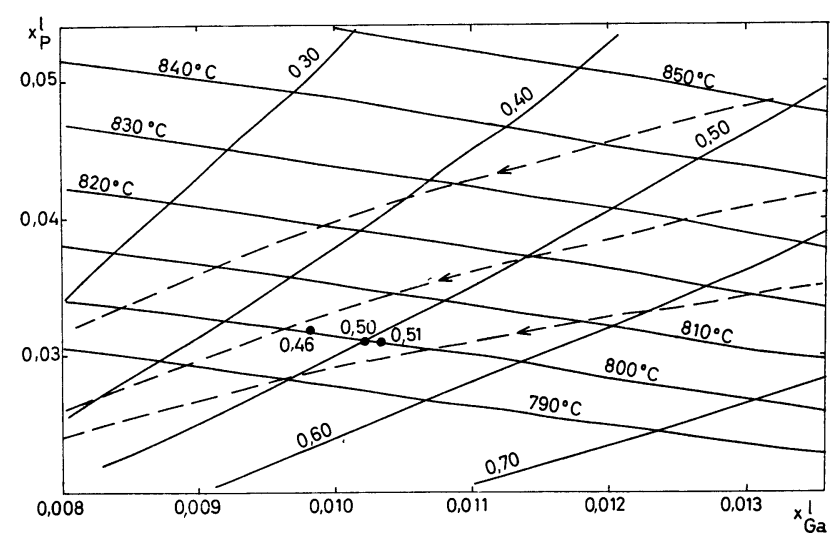

FIG. 2. lignes isosolidus et liquidus calculées au voisinage de la composition $x=0,50 ;-\rightarrow-$ chemins de cristallisation à l'équilibre; . points expérimentaux.

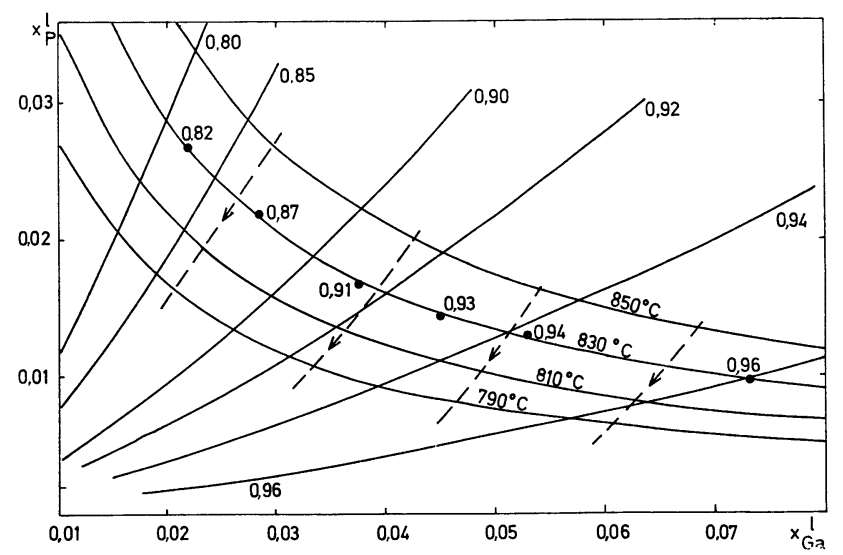

FIG. 3. - lignes isosolidus et liquidus calculées pour les compositions $x \geqslant 0,80 ;-\rightarrow-$ chemins de cristallisation à l'équilibre ; . points expérimentaux. solution liquide est en équilibre avec la solution liquide restante supposée homogène. En se fixant la composition initiale $x_{\mathbf{G a}}^{1_{0}}, x_{\mathrm{P}}^{1_{0}}$ du liquide saturé, l'ensemble des éqs: (1), (2) et (3) permet de prévoir l'évolution de la composition du solide avec la température de cristallisation.

Cette évolution est représentée par les courbes discontinues des figures 2 et 3 , correspondant aux deux régions du diagramme Ga-In-P utiles pour nos expériences d'épitaxie liquide $(\S 2)$. La figure 2 indique que, dans le domaine de température $790-830^{\circ} \mathrm{C}$, une croissance à température lentement variable obtenue par un refroidissement $\Delta T$ de $30^{\circ} \mathrm{C}$ donne un dépôt de composition variable : le calcul précédent prévoit alors une variation de $x=0,5$ à $x=0,4$ sur une épaisseur de $25 \mu \mathrm{m}$. Pour obtenir des couches homogènes sur une épaisseur de plus de $5 \mu \mathrm{m}$, il est donc important de cristalliser à température constante.

Au contraire, pour les couches de composition $x$ supérieure à 0,8 , la figure 3 laisse espérer qu'une technique classique d'épitaxie à température lentement variable permettra l'obtention de dépôts homogènes en épaisseur.

3. Croissance des couches épitaxiques $\mathbf{G a}_{x} \mathbf{I n}_{1-x} \mathbf{P}$ pour $x \simeq 0,50$. - Pour les cristaux massifs une méthode autorisant une cristallisation à température constante a permis d'obtenir des polycristaux relativement homogènes [14-17]. Dans le cas de couches épitaxiques $\mathrm{Ga}_{x} \mathrm{In}_{1-x} \mathrm{P}$ une technique horizontale de croissance en solution à température constante a été décrite par G. B. Stringfellow et coll. [3] et par H. M. Macksey et coll. [18]. La figure 4 représente une illustration schématique de l'appareil et du principe de croissance que nous avons mis en œuvre. Après avoir été saturée à une température $T_{\mathrm{S}}$, la solution est placée dans un léger

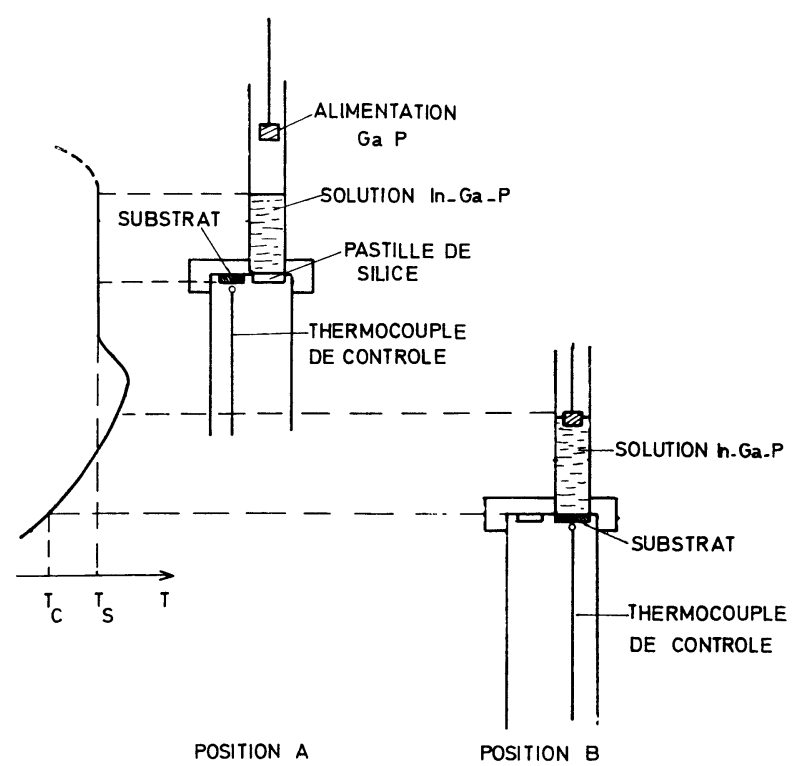

Fig. 4. - Schéma et principe mis au point pour la technique verticale de croissance épitaxique en phase liquide à température constante. 
gradient de température : celui-ci permet, d'une part une sursaturation à l'interface substrat-solution placé au point froid $T_{\mathrm{C}}$ (Fig. 4), et d'autre part une diffusion plus rapide des solutés gallium et phosphore qui alimentent d'une manière continue la solution à partir d'une source de GaP solide. Nous utilisons un appareillage en quartz comportant deux parties excentrées l'une par rapport à l'autre, afin de pouvoir, par la rotation de la partie inférieure, mettre le substrat au contact de la solution au moment voulu. Un rodage plan assure l'étanchéité vis-à-vis du bain liquide. Un four dont le profil est composé d'un palier et d'un gradient de température peut coulisser autour des pièces de quartz afin de pouvoir être positionné en $\mathrm{A}$ ou B (Fig. 4). L'ensemble peut être purgé sous vide et placé sous un courant d'hydrogène purifié.

Nous avons cherché à réaliser des alliages de composition voisine de $x=0,51$. Les substrats de GaAs, orientés suivant la face (111) B, sont polis mécaniquement avec de la pâte diamantée, puis mécanochimiquement à l'aide d'une solution de $\mathrm{NaClO}$. Un léger décapage chimique précède l'expérience.

La croissance est favorisée par une température élevée, mais celle-ci est nécessairement limitée par des considérations de pression de vapeur : la pression de phosphore doit être suffisamment faible pour que la perte en phosphore soit négligeable [19] et ne modifie pas la composition du bain durant la croissance. A $800^{\circ} \mathrm{C}$ cette condition est réalisée.

La composition du liquide utilisé pour la croissance des alliages $\mathrm{Ga}_{\boldsymbol{x}} \mathrm{In}_{1-x} \mathbf{P}$ est obtenue à partir du diagramme de phase de la figure 2 où sont reportés les points expérimentaux obtenus lors des expériences décrites ci-dessous.

Le mélange est préparé en pesant les quantités appropriées d'indium (pureté $6 \mathrm{~N}$ ), de InP et de GaP. Pour obtenir un alliage de composition $x=0,51$ à $800^{\circ} \mathrm{C}$, les quantités initiales sont : $3 \mathrm{~g} \mathrm{~d}$ 'In, $77 \mathrm{mg}$ de InP et $29 \mathrm{mg}$ de GaP. Le système est placé sous vide, puis purgé à l'hydrogène, avant d'être chauffé. Durant deux heures le four est en position A : l'ensemble de la solution se trouve alors dans le palier de température $T_{\mathrm{S}}=805^{\circ} \mathrm{C}$ (Fig. 4). Après cette période d'équilibration où GaP et InP se sont dissous dans l'indium, la source de GaP est mise au contact de la solution pour ajuster la saturation ; par pesée de la quantité dissoute nous obtenons un point expérimental du liquidus (Fig. 2).

Dans une deuxième étape le four est positionné en B : la solution se trouve alors dans un léger gradient de température situé de part et d'autre de la température d'équilibre. A l'extrémité la plus froide, $T_{\mathrm{C}}=800^{\circ} \mathrm{C}$, le substrat est mis au contact de la solution. A l'extrémité la plus chaude, la source de GaP alimente le bain au fur et à mesure qu'il s'appauvrit en gallium et en phosphore. La croissance est arrêtée 30 à 60 min plus tard par le retrait du substrat. Le système est alors refroidi à la température ambiante.
Entre l'étape de saturation et celle de croissance, après avoir retiré la source de $\mathrm{GaP}$, il peut être utile de surchauffer le bain de $10^{\circ} \mathrm{C}$ environ afin de bien dissoudre toutes les cristallites de $\mathrm{GaP}$ et d'InP dans l'indium.

4. Croissance des couches épitaxiques $G a_{x} I_{1-x} P$ pour les compositions $x>\mathbf{0 , 8 0}$. - Les substrats utilisés sont des échantillons de $\mathrm{GaP}$ orientés suivant le plan (111) B. Des dépôts ont été réalisés par la méthode décrite précédemment : typiquement les quantités utilisées pour un alliage $\mathrm{Ga}_{0,90} \mathrm{In}_{0,10} \mathrm{P}$ sont : $3 \mathrm{~g}$ d'In, $39 \mathrm{mg}$ de $\mathrm{GaP}$ et $22 \mathrm{mg}$ de Ga. Cependant la sursaturation de la solution avant la mise au contact du substrat ne s'impose plus, puisqu'un léger décapage in situ de $\mathrm{GaP}$ est acceptable.

Dans le cas des alliages de composition $x>0,80$, le diagramme de la figure 3 permet d'envisager une méthode plus simple de croissance, à température lentement variable, que nous avons réalisée dans un appareillage horizontal classique. Nous avons ainsi obtenu dans la gamme de composition $0,80<x<1$ des dépôts homogènes de $\mathrm{Ga}_{x} \operatorname{In}_{1-x} \mathrm{P}$ par un refroidissement lent de la solution $\left(\Delta T=30^{\circ} \mathrm{C}\right)$, ce qui est impossible pour des dépôts de composition $x \simeq 0,50$. La technique alors retenue pour la mise au contact substrat-solution est le déplacement d'un tiroir portesubstrat par rapport à un creuset en graphite contenant la solution. La solution est tout d'abord chauffée à une température supérieure de $5^{\circ} \mathrm{C}$ à celle prévue par le diagramme d'équilibre afin de permettre une dissolution complète des éléments et une bonne homogénéisation de la solution. La température est ensuite ramenée à celle du liquidus $\left(T_{\mathrm{S}}=830^{\circ} \mathrm{C}\right)$ pendant une demiheure. On amorce alors la descente en température à vitesse programmée $\left(0,33^{\circ} \mathrm{C} / \mathrm{min}\right)$. La mise au contact substrat-solution peut être effectuée soit à la température du liquidus, soit à une température légèrement plus basse $\left(825^{\circ} \mathrm{C}\right)$. A $800^{\circ} \mathrm{C}$ la plaquette est repoussée et l'ensemble est refroidi jusqu'à la température ambiante.

Cette série d'expériences a permis de tracer le liquidus expérimental à $830^{\circ} \mathrm{C}$ et de déterminer l'intersection des lignes iso-solidus avec cette courbe (Fig. 3).

Des dopages à l'azote ont été réalisés dans le cas des compositions $x>0,85$ grâce à un flux d'ammoniac. Le contrôle de ce dopage est effectué en faisant varier la pression partielle d'ammoniac dans l'hydrogène.

5. Caractérisation des couches épitaxiques. - La morphologie de la surface est observée au microscope métallographique. Elle est extrêmement variable pour les alliages déposés sur GaAs et dépend très fortement de l'égalité des paramètres cristallins du substrat et de la couche épitaxique, donc de la composition de la couche. Ainsi la figure $5 a$ présente un aspect de croissance régulière. De larges ondulations apparaissent par contre en $5 b$ : ce phénomène de surfaces ondulées cor- 


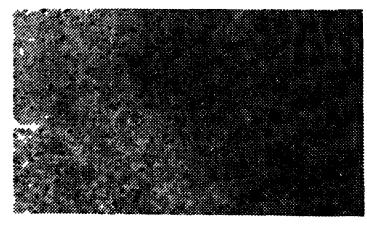

a

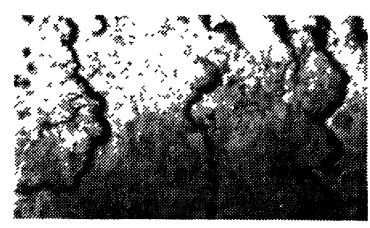

b

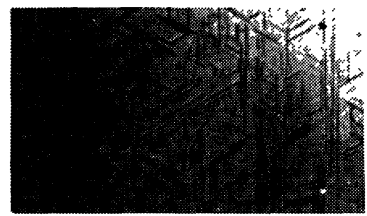

c Ech: $40 \mu$

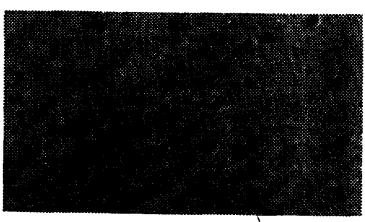

d

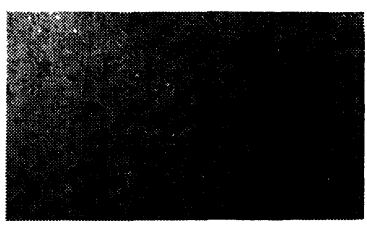

e

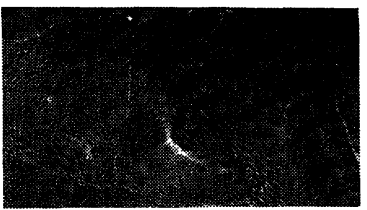

$f$
FIG. 5. - Morphologie des surfaces des couches obtenues :

a) $\mathrm{Ga}_{0,51} \mathrm{In}_{0,49} \mathrm{P} / \mathrm{GaAs}$; d) $\mathrm{Ga}_{0,96} \mathrm{In}_{0,04} \mathrm{P} / \mathrm{GaP}$;

b) $\mathrm{Ga}_{0,50} \mathrm{In}_{0,50} \mathrm{P} / \mathrm{GaAs}$; e) $\mathrm{Ga}_{0,91} \mathrm{In}_{0,09} \mathrm{P} / \mathrm{GaP}$;

c) $\left.\mathrm{Ga}_{0,52} \operatorname{In}_{0,48} \mathrm{P} / \mathrm{GaAs} ; f\right) \mathrm{Ga}_{0,85} \mathrm{In}_{0,15} \mathrm{P} / \mathrm{GaP}$.

respondant à une croissance par chevauchement de gradins a déjà été mentionné pour des dépôts de $\mathrm{Ga}_{x} \mathrm{In}_{1-x} \mathrm{P}$ sur des substrats de GaAs (111) B [20]. Une surface montre après décapage chimique l'apparition de figures d'attaque respectant la symétrie ternaire du plan (111) (Fig. 5c).

Pour des compositions s'éloignant de $x=0,51 \pm 0,01$, l'apparence de la couche se dégrade rapidement ; on observe alors des inclusions d'indium.

Pour les dépôts riches en gallium $(x>0,80)$ la surface de la couche se détériore moins rapidement que précédemment lorsque la composition en indium augmente (Fig. $5 d, e, f$ ). Cette évolution de la qualité cristalline avec l'écart paramétrique entre couche épitaxiale et substrat se traduit par une augmentation de la densité de dislocations observées après révélation chimique : la densité obtenue varie de $10^{5} \mathrm{~cm}^{-2}$ pour $x=1$ (valeur comparable à celle du substrat) à $7 \times 10^{7} \mathrm{~cm}^{-2}$ pour $x=0,90$.

Une croissance pyramidale prédomine pour les échantillons de composition $x$ inférieure à 0,90 (Fig. $5 f$ ) ; dans ce dernier cas la croissance, dirigée suivant l'axe [111], est constituée d'une juxtaposition de petits cristaux plus ou moins désorientés les uns par rapport aux autres. Ceci a été vérifié sur des diagrammes de Laue en retour.

Pour obtenir des dépôts de meilleure qualité cristalline dans la gamme de composition $0,80<x<0,88$ il semble préférable d'après Z $\mathrm{Z}$. I. Alferov et coll. de travailler sur la face (111) A des substrats de GaP [21].

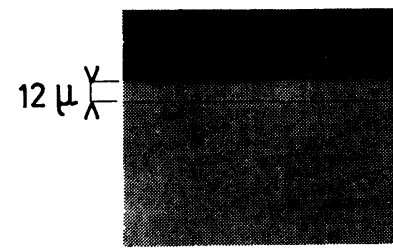

a

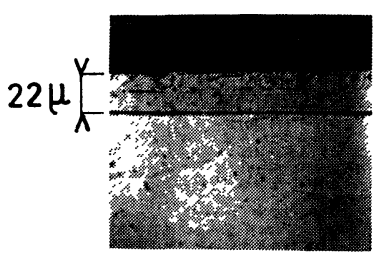

b

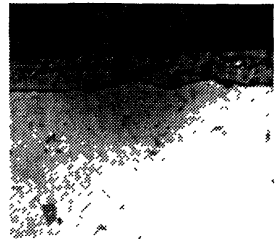

C

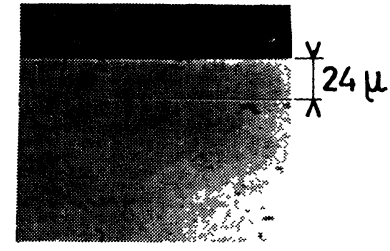

d
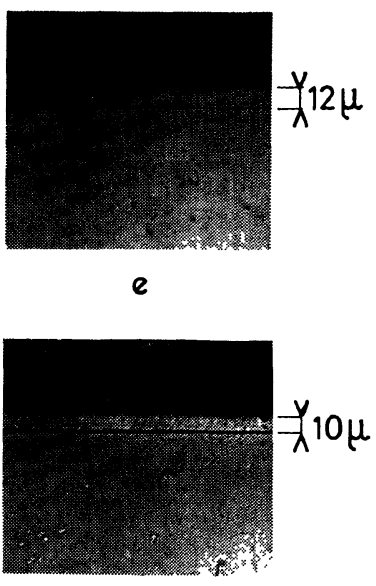

f
FIG. 6. - Plans de clivage des échantillons : $a$ ) $\mathrm{Ga}_{0,51} \mathrm{In}_{0,49} \mathrm{P} / \mathrm{GaAs}$ obtenu avec sursaturation de la solution ; b) $\mathrm{Ga}_{0,50} \mathrm{In}_{0,50} \mathrm{P} / \mathrm{GaAs}$ obtenu avec sursaturation de la solution; c) Couche obtenue sans sursaturation de la solution; d) $\left.\mathrm{Ga}_{0,96} \mathrm{In}_{0,04} \mathrm{P} / \mathrm{GaP} ; e\right) \mathrm{Ga}_{0,91} \mathrm{In}_{0,09} \mathrm{P} / \mathrm{GaP}$; f) $\mathrm{Ga}_{0,85} \operatorname{In}_{0,15} \mathrm{P} / \mathrm{GaP}$.

La figure 6 présente des plans de clivage perpendiculaires au plan de croissance. Une légère attaque chimique permet de révéler l'interface $\mathrm{Ga}_{x} \mathrm{In}_{1-x} \mathrm{P} / \mathrm{GaAs}$ ou $\mathrm{Ga}_{x} \mathrm{In}_{1-x} \mathrm{P} / \mathrm{GaP}$. L'importance du degré de sursaturation de la solution avant la mise au contact du substrat est ainsi mise en évidence. Les dépôts des figures $6 a$ et $6 b$ ont été obtenus en refroidissant le mélange de $5^{\circ} \mathrm{C}$ et $10^{\circ} \mathrm{C}$ avant l'introduction de GaAs. L'interface est rectiligne et le dépôt ne contient pas d'arsenic (Fig. 7a). La figure $6 c$ montre un dépôt réalisé sans sursaturation initiale du mélange $\left(T_{\mathrm{C}}=T_{\mathrm{S}}\right)$ : le substrat est alors légèrement dissous, l'interface n'est plus rectiligne et le dépôt contient de l'arsenic (fig. $7 b$ ) ainsi que des inclusions d'indium. GaAs, n'étant pas en équilibre avec le mélange Ga-In-P, peut se dissoudre au contact du bain ; si la solution est sursaturée, la croissance démarre rapidement sur tout le substrat empêchant sa dissolution.

La vitesse de croissance obtenue est de l'ordre de 20 à $30 \mu \mathrm{m} / \mathrm{h}$. Pour les dépôts riches en $\mathrm{Ga}$ (Fig. $6 d, e, f$ ) la vitesse a tendance à être plus lente, en particulier pour les dépôts réalisés par la méthode horizontale à $T$ variable.

Sur les faces clivées telles qu'elles se présentent sur la figure 6 , nous avons étudié le profil de composition à l'aide d'une microsonde de Castaing. La raie $K \alpha_{1}$ du gallium (témoin $\mathrm{GaAs}$ ou $\mathrm{GaP}$ ), la raie $\mathrm{K} \alpha_{1}$ de l'arsenic 

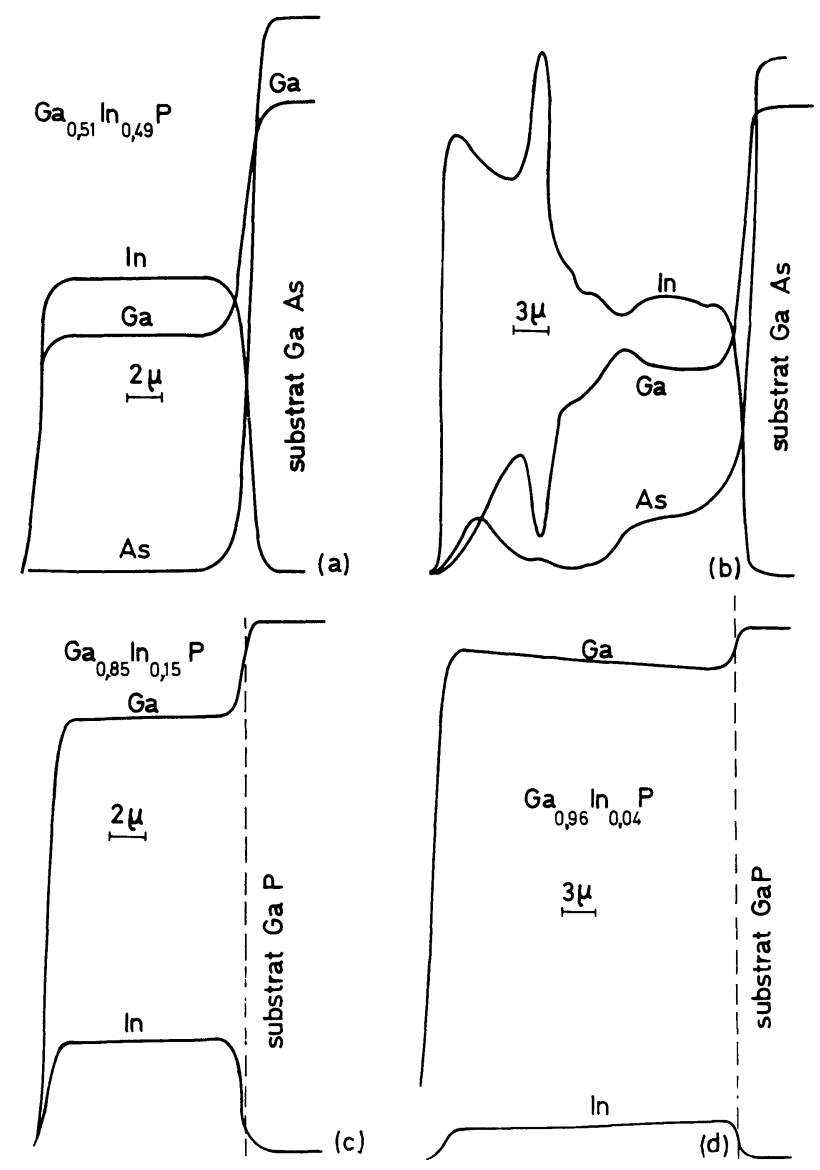

FIG. 7. - Profils de composition mesurés à la microsonde de Castaing : $a$ ) correspond à l'échantillon de la figure $6 a ; b$ ) correspond à l'échantillon de la figure $6 c ; c)$ correspond à l'échanillon de la figure $6 f ; d$ ) correspond à l'échantillon de la figure $6 d$.

(témoin GaAs), la raie $\mathrm{L} \alpha_{1}$ de l'indium (témoin InP) et la raie $\mathrm{K} \alpha_{1}$ du phosphore (témoin InP) sont utilisées pour l'analyse. Les profils sont représentés sur la figure 7 et correspondent aux interfaces $\mathrm{Ga}_{x} \mathrm{In}_{1-x} \mathrm{P} / \mathrm{GaAs}$ et $\mathrm{Ga}_{\boldsymbol{x}} \mathrm{In}_{1-x} \mathrm{P} / \mathrm{GaP}$ des figures $6 a$, $c, d, f$. La composition des échantillons est également déterminée en surface en plusieurs points, ce qui permet de vérifier l'homogénéité de toute la couche : nous obtenons des échantillons homogènes à $1 \%$ atomique absolu près.

Des spectres caractéristiques de photoluminescence à $4,2 \mathrm{~K}$ sont représentés sur les figures 8 et 9 . Pour $x=0,50$, nous obtenons un spectre dont les maxima correspondent à des énergies identiques à celles données par J. Chevallier [22] et H. Kressel et coll. [23]. Le pic de haute énergie est attribué à une recombinaison bande à bande. Le pic de basse énergie prédomine lorsque l'on s'écarte de la composition $x=0,51$ (Fig. 8, ligne discontinue). Il peut être dû, comme le suggèrent W. R. Hitchens et coll. [20], à une compensation partielle due à des défauts associés au désaccord paramétrique. La figure 9 représente le spectre d'un échantillon $x=0,94$ non intentionnellement dopé (ligne discontinue) et celui d'un échantillon dopé à l'azote (ligne continue). Une étude détaillée permet d'identifier ces

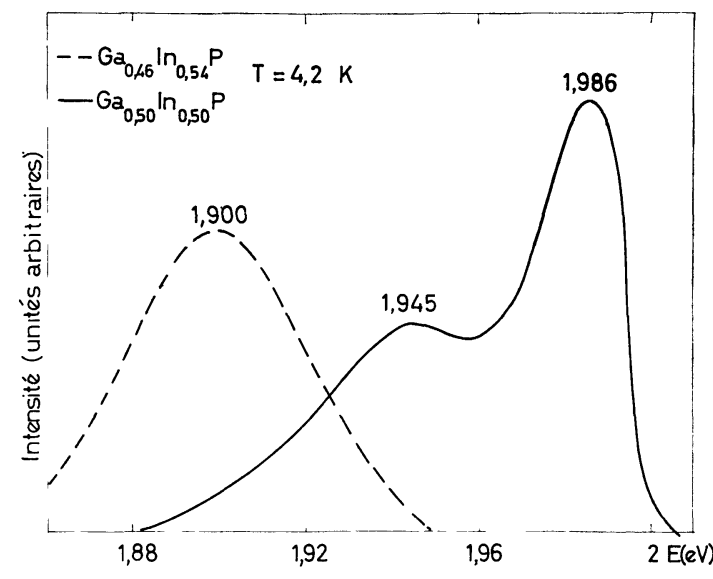

FIG. 8. - Spectres de photoluminescence obtenus à $4,2 \mathrm{~K}$ pour des échantillons $\mathrm{Ga}_{0,50} \mathrm{In}_{0,50} \mathrm{P}$ (ligne continue) et $\mathrm{Ga}_{0,46} \mathrm{In}_{0,54} \mathrm{P}$ (ligne discontinue).

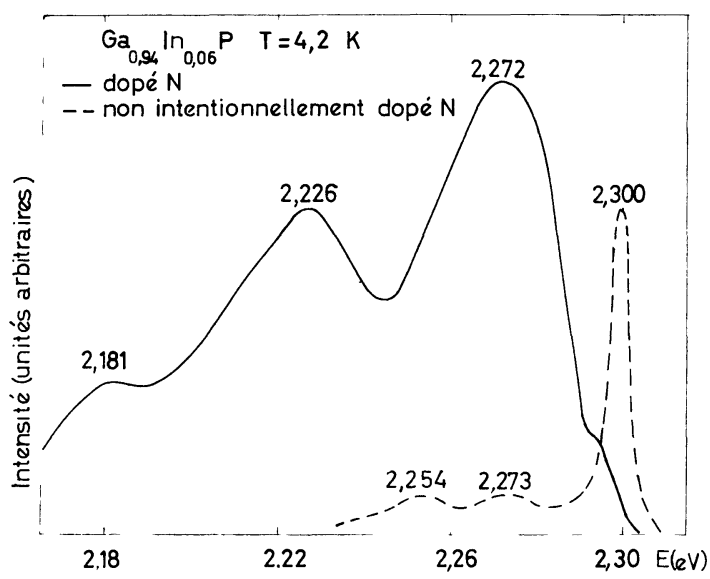

FIG. 9. - Spectres de photoluminescence obtenus à $4,2 \mathrm{~K}$ pour $\mathrm{Ga}_{0,94} \mathrm{In}_{0.06} \mathrm{P}$ dopé à l'azote (ligne continue) et non intentionnellement dopé à l'azote (ligne discontinue).

raies grâce à des mesures de durée de vie (J. Chevallier et $\mathrm{H}$. Mariette, à paraître).

Nous avons montré qu'il s'agissait dans le premier cas d'un exciton lié à un donneur neutre et dans le second d'exciton lié à l'azote.

6. Conclusion. - Partant de la connaissance précise $\mathrm{du}$ diagramme ternaire et utilisant l'hypothèse de solution pseudo-régulière, nous avons développé un modèle de croissance à l'équilibre qui permet de prévoir l'évolution de la composition du solide $\mathrm{Ga}_{x} \mathrm{In}_{1-x} \mathrm{P}$ en fonction de la température. Ce modèle ne rend pas compte exactement des conditions expérimentales car il suppose une vitesse de cristallisation suffisamment faible pour permettre l'homogénéisation instantanée du bain. Il constitue néanmoins une bonne approche puisque nous avons vérifié expérimentalement qu'il était possible, à température variable, de cristalliser des couches homogènes de composition $x$ supérieures à 
0,80 alors que pour $x \simeq 0,50$ une cristallisation à température constante s'imposait. L'énergie d'interaction dans l'alliage conduisant à une meilleure concordance du diagramme théorique avec l'expérience a été calculée : $\Omega_{\mathrm{GaP}-\text { InP }}=3200 \mathrm{cal}$. mole $^{-1}$.

Nous avons développé une méthode de croissance épitaxique en phase liquide à température constante. Celle-ci nous a permis d'obtenir des couches monocristallines homogènes dans le domaine de composition $0,50<x<0,52$ sur support GaAs.

Pour $0,90<x<1$, des couches monocristallines de $\mathrm{Ga}_{x} \mathrm{In}_{1-x} \mathrm{P}$ sur support $\mathrm{GaP}$ ont été préparées ; dans le domaine de composition $0,80<x<0,90$ les dépôts sont également homogènes mais de qualité cristalline moins bien contrôlée.

Les dépôts correspondant aux compositions $x \geqslant 0,85$ ont pu être dopés à l'azote.

Remerciements. - Nous remercions Mme H. Rodot qui nous a initiés à ce travail, $M$. Guillaume pour l'aide qu'il nous a apportée lors du calcul du diagramme de phase, M. Rouy pour les nombreuses mesures effectuées à la microsonde de Castaing, ainsi que les personnes du Laboratoire des Prototypes et de l'Atelier Central de Bellevue qui ont construit les appareillages nécessaires à la réalisation de ce travail.

\section{Bibliographie}

[1] ARCHER, R. J., J. Electron. Mater. 1 (1972) 128.

[2] Alibert, C., Bordure, G., Laugier, A. et Chevallier, J., Phys. Rev. B 6 (1972) 1301.

[3] Stringfellow, G B., Linquist, P. F. et Burmeister, R. A., J. Electron. Mater. 1 (1972) 437.

[4] Lee, M. H., Holonyak N. Jr, Hitchens, W. R., Campbell, J. C. et Altarelli, M., Solid State Commun. 15 (1974) 981

[5] Pitt, G. D., Vyas, M. K. R. et Mabbitt, A. W., Solid State Commun. 14 (1974) 621.

[6] Onton, A. et LoREnTz, M. R., Proc. of the 1970 symposium on GaAs and related compounds, Aachen, Germany (1970) 222

[7] Stringfellow, G. B., J. Appl. Phys. 43 (1972) 3455.

[8] Stringfellow, G. B. et Greene, P. E., J. Phys. Chem. Solids 30 (1969) 1779.

[9] Stringfellow, G. B., J. Electrochem. Soc. 117 (1970) 1301.

[10] Panish, M. B. et Ilegems, M., Progress in Solid State Chemistry Vol. 7, (Pergamon Press New York 1972).

[11] Kajiyama, K., Japan J. Appl. Phys. 10 (1971) 5.

[12] Foster, L. M., J. Electrochem. Soc., Extended Abstract for Spring (1972) Meeting in Houston.
[13] Stringfellow, G B., J. Phys. Chem. Solids 34 (1973) 1749.

[14] Rodot, H., Hruby, A. et Schneider, M., J. Cryst. Growth 3 (1968) 305.

[15] Rodot, H., Horak, J., Rouy, G. et Bourneix, J., $C$. $R$ Hebd. Séan. Acad. Sci. B 269 (1969) 381.

[16] Laugier, A. et Chevallier, J., Phys. Stat. Sol. A 7 (1971) 427.

[17] Macksey, H. M., Holonyak N. Jr, Dupuis, R. O., CampBeLL, J. C. et ZACK, G. W., Appl. Phys. Lett. 19 (1971) 271.

[18] Macksey, H. M., Lee, M. H., Holonyak Jr. N., HitChens, W. R., Dupuis, R. D. et Campbell, J. C., $J$. Appl. Phys. 44 (1973) 5035.

[19] Panish, P. B., J. Chem. Thermodynamics 2 (9170) 319.

[20] Hitchens, W. R., HolonyaK N. Jr, Lee, M. H. et CampBELL, J. C., J. Cryst. Growth 27 (1974) 154

[21] Alferow, Z. I., Konnikov, S. G., Misurnyu, V. A., TreT'JAKow, D. M. et GodLINNiK, T. B., Krist. Techn. 8 (1973) 1029.

[22] Chevallier, J., J. Physique Colloq. 35 (1974) C3-207.

[23] Kressel, H., Nuese, C. J. et Ladany, J., J. Appl. Phys. 44 (1973) 3266. 